\title{
Quantum Geometry on Quantum Spacetime: Distance, Area and Volume Operators
}

\author{
D. Bahns ${ }^{1}$, S. Doplicher ${ }^{2}$, K. Fredenhagen ${ }^{3}$, G. Piacitelli ${ }^{4}$ \\ 1 Courant Research Center Mathematics, University of Göttingen, Bunsenstr. 3-5, 37073 Göttingen, \\ Germany. E-mail: bahns@uni-math.gwdg.de \\ 2 Dipartimento di Matematica, Università degli Studi di Roma "Sapienza", P.le A. Moro 2, 00185 Roma, \\ Italy. E-mail: dopliche@mat.uniroma1.it \\ 3 II. Institut für Theoretische Physik, Universität Hamburg, Luruper Chaussee 149, 22761 Hamburg, \\ Germany. E-mail: klaus.fredenhagen@desy.de \\ 4 SISSA - Via Bonomea 265, 34136 Trieste, Italy. E-mail: gherardo@piacitelli.org
}

Received: 18 May 2010 / Accepted: 26 June 2011

Published online: 27 October 2011 - (C) The Author(s) 2011. This article is published with open access at Springerlink.com

\begin{abstract}
We develop the first steps towards an analysis of geometry on the quantum spacetime proposed in Doplicher et al. (Commun Math Phys 172:187-220, 1995). The homogeneous elements of the universal differential algebra are naturally identified with operators living in tensor powers of Quantum Spacetime; this allows us to compute their spectra. In particular, we consider operators that can be interpreted as distances, areas, 3- and 4-volumes.

The Minkowski distance operator between two independent events is shown to have pure Lebesgue spectrum with infinite multiplicity. The Euclidean distance operator is shown to have spectrum bounded below by a constant of the order of the Planck length. The corresponding statement is proved also for both the space-space and space-time area operators, as well as for the Euclidean length of the vector representing the 3-volume operators. However, the space 3 -volume operator (the time component of that vector) is shown to have spectrum equal to the whole complex plane. All these operators are normal, while the distance operators are also selfadjoint.

The Lorentz invariant spacetime volume operator, representing the 4-volume spanned by five independent events, is shown to be normal. Its spectrum is pure point with a finite distance (of the order of the fourth power of the Planck length) away from the origin.

The mathematical formalism apt to these problems is developed and its relation to a general formulation of Gauge Theories on Quantum Spaces is outlined. As a byproduct, a Hodge Duality between the absolute differential and the Hochschild boundary is pointed out.
\end{abstract}

\section{Introduction and Preliminaries}

The concurrence of the principles of Quantum Mechanics and of Classical General Relativity imposes limits on the joint precision allowed in the measurement of the four spacetime coordinates of an event, as a consequence of the following principle: 
The gravitational field generated by the concentration of energy required by the Heisenberg Uncertainty Principle to localise an event in spacetime should not be so strong to hide the event itself to any distant observer - distant compared to the Planck scale.

These limitations pose no restriction on the precision in the measurement of a single coordinate, ${ }^{1}$ but lead to Spacetime Uncertainty Relations

$$
\Delta q^{0} \cdot \sum_{j=1}^{3} \Delta q^{j} \gtrsim \lambda_{P}^{2} ; \quad \sum_{1 \leq j<k \leq 3} \Delta q^{j} \Delta q^{k} \gtrsim \lambda_{P}^{2},
$$

that were shown to be exactly implemented by Commutation Relations between coordinates, which turn spacetime into Quantum Spacetime [1,4].

More precisely, the four spacetime coordinates of an event are described by four operators which fulfill

$$
\left[q^{\mu}, q^{\nu}\right]=i \lambda_{P}^{2} Q^{\mu \nu}
$$

where $\lambda_{P}$ denotes the Planck length,

$$
\lambda_{P}=\left(\frac{G \hbar}{c^{3}}\right)^{1 / 2} \simeq 1.6 \times 10^{-33} \mathrm{~cm},
$$

hereafter set equal to 1 adopting absolute units, where $\hbar=c=G=1 ; Q$ fulfills the Quantum Conditions

$$
\begin{aligned}
(1 / 4)\left[q^{0}, q^{1}, q^{2}, q^{3}\right]^{2} & =I, \\
{\left[q^{\mu}, q^{\nu}\right]\left[q_{\mu}, q_{\nu}\right] } & =0, \\
{\left[\left[q^{\mu}, q^{\nu}\right], q^{\lambda}\right] } & =0 .
\end{aligned}
$$

Namely, the only full Lorentz invariant constructed with the commutator which is required to be nonzero is the square of the pseudoscalar

$$
\begin{aligned}
{\left[q^{0}, q^{1}, q^{2}, q^{3}\right] } & \equiv \operatorname{det}\left(\begin{array}{cccc}
q^{0} & q^{1} & q^{2} & q^{3} \\
q^{0} & q^{1} & q^{2} & q^{3} \\
q^{0} & q^{1} & q^{2} & q^{3} \\
q^{0} & q^{1} & q^{2} & q^{3}
\end{array}\right) \\
& \equiv \varepsilon_{\mu \nu \lambda \rho} q^{\mu} q^{\nu} q^{\lambda} q^{\rho} \\
& =-(1 / 2) Q^{\mu \nu}(* Q)_{\mu \nu},
\end{aligned}
$$

where

$$
(* Q)_{\mu \nu}=\frac{1}{2} \varepsilon_{\mu \nu \lambda \rho} Q^{\lambda \rho} .
$$

1 This does not conflict with the famous Amati Ciafaloni Veneziano Generalised Uncertainty Relation: all the derivations we are aware of (see e.g. [2,3]) implicitly assume that all space coordinates of the event are measured with uncertainties of the same order of magnitude; in which case they agree with our Spacetime Uncertainty Relations. 
In this model, the $C^{*}$-Algebra of Quantum Spacetime $\mathcal{E}$ is defined as the enveloping $\mathrm{C}^{*}$-Algebra of the Weyl form (selecting the regular representations ${ }^{2}$ ) of the commutation relations between the coordinates:

$$
e^{i \alpha_{\mu} q^{\mu}} e^{i \beta_{\mu} q^{v}}=e^{-(i / 2) \alpha_{\mu} Q^{\mu v} \beta_{v}} e^{i(\alpha+\beta)_{\mu} q^{\mu}} ; \quad \alpha, \beta \in \mathbb{R}^{4} .
$$

The unbounded operators $q^{\mu}$ are affiliated to the $\mathrm{C}^{*}$-Algebra $\mathcal{E}$ and fulfill the desired commutation relations. Poincaré covariance is expressed by an action $\tau$ of the full Poincaré group by automorphisms of $\mathcal{E}$, determined by the property that its canonical extension to the $q$ 's fulfill

$$
\tau_{L}(q)=L^{-1}(q)
$$

The $\mathrm{C}^{*}$-Algebra $\mathcal{E}$ turns out to be the $\mathrm{C}^{*}$-Algebra of continuous functions vanishing at infinity from a manifold $\Sigma$ to the $C^{*}$-Algebra of compact operators on the separable infinite dimensional Hilbert space.

Here $\Sigma$ is the (maximal) joint spectrum of the commutators, which is the manifold of the real antisymmetric two-tensors fulfilling the constraints imposed by the above quantum conditions; namely, specifying such a tensor by its electric and magnetic components $\vec{e}, \vec{m}, \vec{e}^{2}=\vec{m}^{2}, \vec{e} \cdot \vec{m}= \pm 1$. Thus $\Sigma$ can be identified with the full Lorentz orbit of the standard symplectic form in four dimensions, that is $\Sigma$ is the union of two connected components, each homeomorphic to $S L(2, \mathbb{C}) / \mathbb{C}_{*}$, or to the tangent manifold $T S^{2}$ to the unit sphere in three dimensions. If $\vec{e}= \pm \vec{m}$ they must be of length one, and span the base $\Sigma^{(1)}$ of $\Sigma$. Thus $\Sigma$ can be viewed as $T \Sigma^{(1)}$.

If we choose $\sigma=(\vec{e}, \vec{m})$ in $\Sigma^{(1)}$ with $\vec{e}=\vec{m}=(1,0,0)$, the corresponding irreducible representation of the operators $q^{\mu}$ can be realised on the Hilbert space $H \otimes H$, with $H=L^{2}(\mathbb{R})$ by

$$
\begin{aligned}
& q^{0}=Q \otimes I, \\
& q^{1}=P \otimes I, \\
& q^{2}=I \otimes Q, \\
& q^{3}=I \otimes P,
\end{aligned}
$$

where $Q$ is the operator of multiplication by $s$ in $H=L^{2}(\mathbb{R}, d s)$ and $P$ is the operator $\frac{1}{i} \frac{\partial}{\partial s}$ on $H$.

Regarding the interpretation of the operators $q_{\mu}$ we recall that in ordinary quantum field theory, the physical meaning of spacetime coordinates is to specify regions on which the observables of the theory, which are expressed in terms of quantum fields, are localized. This is still true in quantum field theory on quantum spacetime, with the only variant that in this case, the joint effect of Heisenberg's principle and general relativity are taken into account by noncommutativity, cf. [1]. In this sense, the noncommutative geometry of the underlying quantum spacetime has the same status as classical geometry (Minkowski space) in ordinary quantum field theory, and in both cases, the coordinates merely describe the underlying geometry.

\footnotetext{
2 An irreducible representation of (1.2) is regular if and only if it generates an irreducible representation of $\mathcal{E}$, or, by the Dixmier - Nelson Theorem, if and only if the sum of the squares of the $q^{\mu}$ is essentially selfadjoint. In this paper we will deal only with such representations.
} 
We now examine the Minkowski square operator $q^{\mu} q_{\mu}$ in Quantum spacetime. Since it is a full Lorentz scalar, it can be computed at a fixed point $\sigma$ in $\Sigma$. Hence, choosing $\sigma$ as here above, we can compute $q^{\mu} q_{\mu}$ using that particular irreducible representation as

$$
q^{\mu} q_{\mu}=\left(Q^{2}-P^{2}\right) \otimes I-I \otimes\left(Q^{2}+P^{2}\right) .
$$

While $Q^{2}+P^{2}$ has pure point spectrum, equal to $2 n+1, n=0,1,2, \ldots,(1 / 2)\left(P^{2}-Q^{2}\right)$ is the Hamiltonian of the anti-harmonic oscillator, which is known to be unitarily equivalent to the direct sum of two copies of $Q$ (or of $P$ ). For instance, a unitary $U$ changing $P \oplus P$ to $(1 / 2)\left(Q^{2}-P^{2}\right)$ by its adjoint action can be given as

$$
\left(U\left(\psi_{1} \oplus \psi_{2}\right)\right)(s)=e^{(i / 2)\left(-i \frac{\partial}{\partial s}\right)^{2}} e^{(i / 4) s^{2}}|s|^{-1 / 2}\left(\theta(s) \psi_{1}(\ln |s|)+\theta(-s) \psi_{2}(\ln |s|)\right)
$$

with the Heaviside function $\theta$. Now $a P+b I$ is unitarily equivalent to $P$ and to $Q$ for any real $a, b$, with $a$ nonzero; therefore $q^{\mu} q_{\mu}$ is unitarily equivalent to the direct sum of infinitely many copies of $Q$, in any irreducible representation, hence in any representation of $\mathcal{E}$.

The Euclidean square operator $\Sigma q_{\mu}^{2}$ has been analysed in [1]. In the above representation, it agrees with the operator,

$$
\left(P^{2}+Q^{2}\right) \otimes I+I \otimes\left(P^{2}+Q^{2}\right),
$$

with pure point spectrum whose minimum is 2 , corresponding to a state optimally localised around the origin. It was shown there that this is the minimum of the spectrum over all representations, and can be attained only in pure states in irreducible representations associated to points in the base $\Sigma^{(1)}$ of $\Sigma$, or in states obtained from them by integration with a regular probability measure on $\Sigma^{(1)}$.

These statements will extend immediately to the Minkowskian respectively Euclidean distance operators between two independent events.

In Quantum Mechanics the observables for the system composed of independent subsystems are described by tensor products of observables for the subsystems. This suggests to describe the algebra of coordinates for $n$ independent spacetime events as the tensor product $\mathcal{E} \otimes \mathcal{E} \otimes \cdots \otimes \mathcal{E}$ ( $n$ times). Thus the coordinate operators for the $j^{\text {th }}$ event are

$$
q_{j}=I \otimes I \otimes \ldots I \otimes q \otimes I \otimes \cdots \otimes I,\left(j^{\text {th }} \text { place }\right) .
$$

It is however more natural to use not the standard tensor product over the complex numbers, but the $\mathcal{Z}$-module tensor product, where $\mathcal{Z}$ denotes the centre of the multiplier algebra of $\mathcal{E}$, that is the algebra of continuous bounded complex functions on $\Sigma$. This amounts to require that the commutators of the different spacetime components of $q_{j}$ are independent of $j$, so that

$$
\left[q_{j}^{\mu}, q_{k}^{v}\right]=i Q^{\mu v} \delta_{j, k},
$$

or, in the language of the next section, that

$$
d Q=0 .
$$

This choice has been discussed and motivated in [5], and implies in particular that the normalised difference variables

$$
\frac{1}{\sqrt{2}}\left(q_{j}-q_{k}\right)
$$


for distinct $j, k$ obey the same commutation relations (1.1), and commute with the barycentre coordinates.

In particular, the Minkowski, respectively Euclidean, distance operators, evaluated with these normalised difference variables for two independent events, will have exactly the same spectral properties as above described for the $q$ s themselves, so that, in absolute units,

$$
\sum_{\mu=0}^{3}\left(q_{j}^{\mu}-q_{k}^{\mu}\right)^{2} \gtrsim 4 .
$$

In the next section we will briefly discuss a variant of the Universal Differential Calculus where this formalism fits best, and the two distinct relevant algebraic structures. This is the natural mathematical ground for a general formulation of Gauge Theories on Quantum Spaces, briefly touched upon in the subsequent section.

In the course of the discussion we will present a novel pairing between differential forms, the q-pairing, which yields a duality between the exterior differential and the Hochschild boundary, which makes of the latter a codifferential.

In this formalism, the exterior products of the $d q$ 's will describe precisely the operators for the area, volume, four-volume spanned by respectively 3, 4 or 5 points describing independent events.

The spectrum of each of these operators, however, has to be defined relative to another structure of *-Algebra, namely viewing the differential forms as operators affiliated to the $\mathrm{C}^{*}$-Algebra direct sum of the $\mathrm{C}^{*}$-algebraic $\mathcal{Z}$-module tensor powers of $\mathcal{E}$.

A similar distinction of structures underlies the usual differential calculus.

The spectra of these operators will be fully computed in the last section. The results confirm what might be anticipated on intuitive grounds, based on the uncertainty relations: if one space coordinate has a very small uncertainty $a$, then at least one other space coordinate and the time coordinate must have an uncertainty $b$ such that $a b \gtrsim 1$; this suggests that the space - space and the space - time areas ought to be bounded below by 1 , as well as the spacetime volume; while the space 3-volume (the time component of the vector representing the 3 -volume operators) might be arbitrarily small, since, in the above situation, the third space coordinate might well have an uncertainty of the same order $a$, so that the product of the three space uncertainties has the same arbitrarily small order; while the further product with the time uncertainty, of order $b$, is again at least of order one. These arguments could be cast into a mathematical proof that the product of the four spacetime uncertainties must be bounded below by order one as a consequence of (1.1).

It is worth noting that the restrictions we find here upon the spectra of the distance, area, and volume operators are just the minimal restrictions, imposed merely by the underlying geometry of our model of quantum Spacetime. In a realistic theory, the restrictions coming from dynamics together with the expected interconnections of spacetime and fields [6] might well impose tighter limitations.

\section{Independent Events and the Universal Differential Calculus}

Let $A$ be an associative algebra with unit over $\mathbb{C}$, obtained e.g. by adding the unit to an algebra $A_{0}$. Any tensor power $\Lambda^{n}(A):=A^{\otimes(n+1)}$ of $A$ over $\mathbb{C}$ can be viewed as an $A$-bimodule (using the product in $A$ on the first, resp. last factor), and the direct sum

$$
\Lambda(A)=\bigoplus_{n=0}^{\infty} \Lambda^{n}(A)
$$


can be viewed as the $A$-bimodule tensor algebra, ${ }^{3}$ so that, in $\Lambda(A)$,

$$
\left(a_{0} \otimes \cdots \otimes a_{n}\right) \cdot\left(b_{0} \otimes \cdots \otimes b_{m}\right)=a_{0} \otimes \cdots \otimes a_{n} b_{0} \otimes \cdots \otimes b_{m} .
$$

The exterior differential is defined on $\Lambda(A)$ by

$$
d\left(a_{0} \otimes \cdots \otimes a_{n}\right)=\sum_{k=0}^{n+1}(-1)^{k} a_{0} \otimes \cdots \otimes a_{k-1} \otimes I \otimes a_{k} \otimes \cdots \otimes a_{n} .
$$

As usual $d$ is a graded differential, i.e., if $\phi \in \Lambda^{n}(A), \psi \in \Lambda(A)$, we have

$$
\begin{aligned}
d^{2} & =0 ; \\
d(\phi \psi) & =(d \phi) \psi+(-1)^{n} \phi d \psi .
\end{aligned}
$$

Note that $A=\Lambda^{0}(A) \subset \Lambda(A)$, and the $d$-stable subalgebra $\Omega(A)$ of $\Lambda(A)$ generated by $A$ is the universal differential algebra (or the universal differential algebra of $A_{0}$ following $[8, \S 1 . \alpha])$.

Observe that $\Omega(A)$ defined as above coincides with the usual notion of the universal differential algebra $\bigoplus_{n \geq 0} \Omega^{n}(A)$ with $\Omega^{0}(A):=A$, and

$$
\Omega^{n}(A):=\Omega^{1}(A) \otimes_{A} \cdots \otimes_{A} \Omega^{1}(A) \quad(n \text { times }) .
$$

For $n=1,2, \ldots$, consider the multiplication maps $m_{k}: \Lambda^{n+1}(A) \rightarrow \Lambda^{n}(A)$ which linearly extend

$$
m_{k}\left(a_{0} \otimes \cdots \otimes a_{n}\right):=a_{0} \otimes \cdots \otimes a_{k} a_{k+1} \otimes \cdots \otimes a_{n},
$$

where $k=0, \ldots, n-1$. Then $\Omega^{n}(A)$ coincides with the intersection of the kernels of all multiplication maps [9].

On $\Lambda(A)$, we define an $A$-valued pairing, called the q-pairing, by linearly extending

$$
\left\langle a_{0} \otimes \cdots \otimes a_{n}, b_{0} \otimes \cdots \otimes b_{m}\right\rangle:=\delta_{n, m} a_{0} b_{0} \ldots a_{n} b_{n} .
$$

Observe that for any $a, b \in A, \omega \in \Lambda^{n}(A), \phi \in \Lambda^{m}(A)$, we have

$$
\langle a \omega, \phi b\rangle=a\langle\omega, \phi\rangle b .
$$

Moreover,

$$
\begin{aligned}
& \langle d a \omega, d b \phi\rangle=[a, b]\langle\omega, \phi\rangle, \\
& \langle\omega d a, \phi d b\rangle=\langle\omega, \phi\rangle[a, b],
\end{aligned}
$$

for all $a, b \in A, \omega, \phi \in \Lambda^{n}(A)$. It follows that, when restricted to $\Omega(A)$, the $q$-pairing has some additional properties: for any $a_{i}, b_{i}, a, b \in A$, and $\omega, d \psi \in \Omega^{n}(A)$, and $\phi, \lambda \in \Omega^{m}(A)$, we have

$$
\begin{aligned}
\left\langle d a_{1} \ldots d a_{n}, d b_{1} \ldots d b_{n}\right\rangle & =\left[a_{1}, b_{1}\right] \ldots\left[a_{n}, b_{n}\right], \\
\langle\omega \phi,(d \psi) \lambda\rangle & =\langle\omega, d \psi\rangle\langle\phi, \lambda\rangle \\
\langle\lambda d \psi, \phi \omega\rangle & =\langle\lambda, \phi\rangle\langle d \psi, \omega\rangle .
\end{aligned}
$$

\footnotetext{
3 For closely related notions, as the free product of two algebras, see [7].
} 
The $A$-valued pairing $\langle\cdot, \cdot\rangle$ turns into an interesting $\mathbb{C}$ - valued pairing by composition with a trace $\tau$. Namely, let $\tau$ be a complex valued linear map defined on a two sided ideal $J$, such that

$$
\tau(a b)=\tau(b a), \quad a \in A, b \in J ;
$$

$\tau$ is faithful if $a$ in $A$ fulfills $\tau(a b)=0$ for all $b$ in $J$ only if $a=0$.

If $\Lambda_{J}(A)$ denotes the differential ideal in $\Lambda(A)$ generated by $J$, we have that $\Lambda_{J}(A)$ is the span of elements $a_{0} \otimes \cdots \otimes a_{n}$, where $a_{j} \in J$ for at least one $j$, and

$$
\langle\phi, \psi\rangle \in J, \quad \phi \in \Lambda(A), \psi \in \Lambda_{J}(A) .
$$

Let $\delta$ denote the Hochschild boundary defined by

$$
\begin{aligned}
\delta\left(a_{0} \otimes \cdots \otimes a_{n}\right)= & \sum_{k=0}^{n-1}(-1)^{k} a_{0} \otimes \cdots \otimes a_{k-1} \otimes a_{k} a_{k+1} \otimes a_{k+2} \otimes \cdots \otimes a_{n} \\
& +(-1)^{n} a_{n} a_{0} \otimes \cdots \otimes a_{n-1} .
\end{aligned}
$$

Then we have

Proposition 2.1. The Hochschild boundary is a Hodge dual of the differential for the pairing $\tau(\langle\cdot, \cdot\rangle)$, namely

$$
\tau(\langle\delta \omega, \phi\rangle)=\tau(\langle\omega, d \phi\rangle), \omega, \phi \in \Lambda(A) .
$$

Proof. A routine computation.

Thus the associated Laplacian is $(d+\delta)^{2}=d \delta+\delta d$; this operator, and the associated Hodge theory, has been studied by Cuntz and Quillen in the context of Cyclic cohomology [7].

A similar conclusion as above might be drawn using, in place of a trace $\tau$, the universal trace, namely the quotient map of $A$ modulo the vector subspace spanned by the commutators [10].

The pairing discussed here reduces exactly to zero in case of a commutative algebra, so it seems to describe an alternative to the classical formalism, possibly valid only in the purely quantum picture, rather than a deformation of the classical calculus, which reduces to it in the limit where the relevant parameter, as the Planck length, is neglected. This property is however fulfilled, in the case of the model of Quantum Spacetime described in the Introduction, by another pairing, that we will mention here below.

Note first that the above formalism can be applied to $\mathcal{E}$, or to $\mathcal{E}$ embedded in its multiplier algebra with centre $\mathcal{Z}$; we will actually use it also for the affiliated unbounded operators as $q$. But it will be important for our application to use everywhere in the above formalism the $\mathcal{Z}$-module tensor product, as anticipated in the Introduction, that is $d Q=0$. We will still write $\Lambda_{n}(M \mathcal{E})$ for the $n^{\text {th }} \mathcal{Z}$ - module tensor product.

In the language of the universal differential calculus, the difference operator for coordinates of two independent events as discussed in the previous section, i.e. $q_{2}-q_{1}=$ $1 \otimes q-q \otimes 1$ is given as $d q$. Observe that this order makes sense since on a (commutative) vector space, $q_{2}-q_{1}$ is the vector that connects $q_{1}$ with $q_{2}$, so it has the same sign as the tangent vector of a straight curve from $q_{1}$ to $q_{2}$. Furthermore, the geometric operators describing area, volume and spacetime volume on Quantum Spacetime spanned by the independent points will be described by the exterior products of the $d q$ 's, evaluated in $\Lambda(M \mathcal{E})$. 
In this setting, we can introduce other pairings $(\cdot, \cdot)$ on $\Omega(\mathcal{E})$ by choosing suitable elements $\mu$ in $\Lambda(\mathcal{E})$, and setting

$$
(\phi, \psi)=\tau(\langle\phi \psi, \mu\rangle) \delta_{n, m}, \quad \phi \in \Omega_{n}(\mathcal{E}), \psi \in \Omega_{m}(\mathcal{E}) .
$$

Using the product of differential forms in $\Lambda(\mathcal{E})$, we now consider a particular choice of $\mu$ which has a natural interpretation as a metric form. To this end, first observe that the $v$-component of spacetime derivations on Quantum Spacetime is given by iAd $\tilde{q}_{\nu}$, where $\tilde{q}_{v}=\left(Q^{-1} q\right)_{\nu}$. Now consider

$$
\mu:=\sum_{n}\left(d \tilde{q}^{\left[\mu_{1}\right.} d \tilde{q}^{\mu_{2}} \ldots d \tilde{q}^{\left.\mu_{n}\right]}\right)\left(d \tilde{q}_{\left[\mu_{1}\right.} d \tilde{q}_{\mu_{2}} \ldots d \tilde{q}_{\left.\mu_{n}\right]}\right)
$$

with totally antisymmetrized products in each of the brackets and Lorentz contraction of upper and lower indices. As we shall see in Sect. 4, this metric operator, $\mu=$ $\sum_{n}(d \tilde{q} \wedge \cdots \wedge d \tilde{q})(d \tilde{q} \wedge \cdots \wedge d \tilde{q})$ in the notation adopted there, can be understood in terms of distance, area and volume operators.

We then find for the corresponding pairing

$$
\left(a_{0} d a_{1} \ldots d a_{n}, d b_{1} \ldots d b_{n} b_{n+1}\right)=\tau\left(a_{0} \partial_{\left[\mu_{1}\right.} a_{1} \ldots \partial_{\left.\mu_{n}\right]} a_{n} \partial^{\left[\mu_{1}\right.} b_{1} \ldots \partial^{\left.\mu_{n}\right]} b_{n} b_{n+1}\right),
$$

which justifies the interpretation of $\mu$ as a metric form.

\section{Connection and Parallel Transport}

In order to introduce gauge theories on noncommutative spaces, one usually starts from modules, interpreted as spaces of sections of a vector bundle with a noncommutative base. Connections for the universal differential calculus can be introduced as covariant derivatives, provided the module is projective. ${ }^{4}$ We also introduce a concept of parallel transport, and by using the q-pairing, extended to module valued forms, we construct a linear map from the algebra to the endomorphisms of the module. This map corresponds to the transition from coordinates to covariant coordinates as introduced in [12] in a somewhat different context. Similar to the discussion there, the curvature of this map turns out to be related to the curvature of the connection. The map can be used to construct gauge invariant local functionals ("local observables") of the theory. The exposition here extends an earlier publication [13].

Let $H$ be a right module over $A$. We set

$$
\Lambda^{n}(A, H):=H \otimes_{A} \Lambda^{n}(A)=H \otimes A^{\otimes n},
$$

which is a right $A$-module. For $\sigma \in \Lambda^{n}(A, H), \omega \in \Lambda^{m}(A)$, we have $\sigma \otimes_{A} \omega \in$ $\Lambda^{n+m}(A, H)$, so

$$
\Lambda(A, H):=\bigoplus_{n=0}^{\infty} \Lambda^{n}(A, H)
$$

\footnotetext{
${ }^{4}$ We are grateful to Jochen Zahn for drawing our attention to [11, Prop 8.3].
} 
is a right $\Lambda(A)$-module with respect to the action $\sigma \omega:=\sigma \otimes_{A} \omega$. As on $\Lambda^{n}(A)$, we have the multiplication maps $m_{k}: \Lambda^{n}(A, H) \rightarrow \Lambda^{n-1}(A, H)$ for $k=0, \ldots, n-1$, given by linearly extending $\left(\sigma \in H, a_{j} \in A\right)$

$$
\begin{aligned}
& m_{0}\left(\sigma \otimes \bigotimes_{i=1}^{n} a_{i}\right):=\sigma a_{1} \otimes \bigotimes_{i=2}^{n} a_{i} \\
& m_{k}\left(\sigma \otimes \bigotimes_{i=1}^{n} a_{i}\right):=\sigma \otimes a_{1} \otimes \cdots \otimes a_{k} a_{k+1} \otimes a_{n} \quad \text { for } 1 \leq k \leq n-1
\end{aligned}
$$

The q-pairing has a straightforward extension to a pairing of $\Lambda^{n}(A, H)$ with $\Lambda^{n}(A)$ with values in $H$, by

$$
\left\langle\sigma \otimes a_{1} \otimes \cdots \otimes a_{n}, b_{0} \otimes \cdots \otimes b_{n}\right\rangle:=\sigma b_{0} \prod_{i=1}^{n} a_{i} b_{i} .
$$

We now consider the set of $H$-valued (universal) $n$-forms

$$
\Omega^{n}(A, H):=H \otimes_{A} \Omega^{n}(A),
$$

which is a right $A$-module. For $\sigma \in \Omega^{n}(A, H), \omega \in \Omega^{m}(A)$, we have $\sigma \otimes_{A} \omega \in$ $\Omega^{n+m}(A, H)$, so

$$
\Omega(A, H):=\bigoplus_{n=0}^{\infty} \Omega^{n}(A, H)
$$

is an $\Omega(A)$-module with respect to the action $\sigma \omega:=\sigma \otimes_{A} \omega$. In particular, we can identify $\Omega^{1}(A, H) \otimes_{A} \Omega^{n}(A)$ with $\Omega^{n+1}(A, H)$.

Once again, $\Omega(A, H)$ is the submodule of $\Lambda(A, H)$ which is given by the intersection of the kernels of the multiplication maps $m_{k}$.

A universal covariant differential (a universal connection) on $H$ is a linear map

$$
D: H \rightarrow \Omega^{1}(A, H)
$$

satisfying the Leibniz rule

$$
D(\sigma a)=(D \sigma) a+\sigma d a
$$

for all $\sigma \in H, a \in A$.

Observe that a right $H$-module must be projective in order to admit a universal connection [11, Prop. 8.3].

If $D$ is a universal connection, it has an extension (still denoted by $D$ ) to $\Omega(A, H)$, which is uniquely fixed by the requirement

$$
D(\sigma \alpha):=(D \sigma) \alpha+\sigma d \alpha
$$

for all $\sigma \in H, \alpha \in \Omega(A)$. Recall here that $\sigma \alpha=\sigma \otimes_{A} \alpha$, and that $(D \sigma) \alpha=(D \sigma) \otimes_{A} \alpha$, and that we have $\Omega^{m}(A, H) \otimes_{A} \Omega^{n}(A)=\Omega^{m+n}(A, H)$. For $\sigma \in \Omega^{n}(A, H)$, and $\alpha \in \Omega(A)$, we then have the graded Leibniz rule

$$
D(\sigma \alpha):=(D \sigma) \alpha+(-1)^{n} \sigma d \alpha .
$$


We will now show that the map $D^{2}: \Omega(A, H) \rightarrow \Omega(A, H)$ is a right $\Omega(A)$-module homomorphism with $D^{2}: \Omega^{n}(A, H) \rightarrow \Omega^{n+2}(A, H)$.

To that end, let $\sigma \in \Omega^{n}(A, H)$ and $\psi \in \Omega(A)$. Then from the graded Leibniz' rule and $d^{2}=0$ we get

$$
\begin{aligned}
D^{2}(\sigma \psi) & =D\left((D \sigma) \psi+(-1)^{n} \sigma d \psi\right) \\
& =\left(D^{2} \sigma\right) \psi+(-1)^{n+1}(D \sigma) d \psi+(-1)^{n}(D \sigma) d \psi \\
& =\left(D^{2} \sigma\right) \psi .
\end{aligned}
$$

By analogy with differential geometry, $F:=D^{2}$ is called the curvature of the connection $D$.

Making use of the inclusion of $\Omega^{n}$ in $\Lambda^{n}$ and the $q$-pairing, we now introduce two new concepts: the parallel transport associated to covariant derivative, and a right module map which generalises the notion of the covariant coordinates introduced in [12].

Let $S: H \rightarrow \Lambda^{1}(A, H)$ be a linear map, then we associate a linear map $U: H \rightarrow$ $\Lambda^{1}(A, H)$ to $S$ by

$$
U(\sigma):=S \sigma+\sigma \otimes 1 .
$$

Given a universal connection $D: H \rightarrow \Omega^{1}(A, H)$, the linear map $U: H \rightarrow$ $\Lambda^{1}(A, H)$ associated with $D$ is called the parallel transport (along $D$ ).

A related notion of parallel transport has been introduced in the context of lattice gauge theory $[14,15]$

Proposition 3.1. The linear map $U$ associated to a linear map $S: H \rightarrow \Lambda^{1}(A, H)$ in the sense of (3.21) is a right module map if and only $S$ satisfies the Leibniz rule w.r.t. the universal calculus.

Observe that we do not assume that $S$ takes values in $\Omega^{1}(A, H)$.

Proof. Let $S$ satisfy the Leibniz rule, then for $\sigma \in H$, and $a \in A$, we have

$$
\begin{aligned}
U(\sigma a) & =S(\sigma a)+\sigma a \otimes 1=(S \sigma) a+\sigma d a+\sigma a \otimes 1 \\
& =(U \sigma-\sigma \otimes 1) a+\sigma(1 \otimes a-a \otimes 1)+\sigma a \otimes 1 \\
& =(U \sigma) a .
\end{aligned}
$$

On the other hand, let $U$ be a right module map, then $S(\sigma):=U(\sigma)-\sigma \otimes 1$ satisfies the Leibniz rule w.r.t. the universal calculus,

$$
S(\sigma a)=U(\sigma) a-\sigma a \otimes 1=(S(\sigma)+\sigma \otimes 1) a-\sigma a \otimes 1=S(\sigma) a+\sigma d a .
$$

If $D$ is a universal connection, the parallel transport $U$ along $D$ is a right module map (by the proposition above) and moreover satisfies $m_{0} \circ U=1$, since $D$ takes values in $\Omega^{1}(A, H)$, so that $m_{0} \circ D=0$ whence $m_{0} \circ U(\sigma)=m_{0}(\sigma \otimes 1)=\sigma$. Conversely, if $U$ is a right module map that satisfies $m_{0} \circ U=1$, then $S:=U-\cdot \otimes 1$ is a universal connection.

It follows that the parallel transport splits the exact sequence

$$
0 \longrightarrow \Omega^{1}(A, H) \hookrightarrow H \otimes A \stackrel{m_{0}}{\longrightarrow} H \longrightarrow 0 .
$$


This splitting map was used to prove that a module admitting a universal connection must be projective in [11, Prop. 8.3].

Using the notion of the parallel transport, we now extend $D$ to all of $\Lambda(A, H)$ in the same spirit as in the definition of the exterior differential on $\Lambda(A)$ in the previous section.

Definition 3.2. On $\Lambda^{n}(A, H)$, the covariant differential is defined, as a linear map into $\Lambda^{n+1}(A, H)$, by

$$
D \sigma \otimes \bigotimes_{i=1}^{n} a_{i}=U \sigma \otimes \bigotimes_{i=1}^{n} a_{i}+\sum_{k=1}^{n+1}(-1)^{k} \sigma \otimes \bigotimes_{i=1}^{k-1} a_{i} \otimes 1 \otimes \bigotimes_{i=k}^{n} a_{i} .
$$

The map defined above satisfies the graded Leibniz rule,

$$
D(\sigma \psi)=(D \sigma) \psi+(-1)^{n} \sigma d \psi, \sigma \in \Lambda^{n}(A, H), \psi \in \Lambda(A) .
$$

To see this, it suffices to prove the proposition for $n=0$. Let $\psi=\bigotimes_{i=0}^{m} a_{i}$. Then $\sigma \psi=\sigma a_{0} \otimes \bigotimes_{i=1}^{m} a_{i}$ and

$$
D(\sigma \psi)=U \sigma a_{0} \otimes \bigotimes_{i=1}^{m} a_{i}+\sum_{k=1}^{m+1}(-1)^{k} \sigma a_{0} \otimes \bigotimes_{i=1}^{k-1} a_{i} \otimes 1 \otimes \bigotimes_{i=k}^{m} a_{i}
$$

On the other hand,

$$
\begin{aligned}
(D \sigma) \psi & =(U \sigma-\sigma \otimes 1) \psi \\
& =U \sigma a_{0} \otimes \bigotimes_{i=1}^{m} a_{i}-\sigma \otimes \bigotimes_{i=0}^{m} a_{i},
\end{aligned}
$$

hence

$$
D(\sigma \psi)-(D \sigma) \psi=\sigma d \psi
$$

which proves the assertion.

By the same argument used for proving (3.20), we see that $D^{2}: \Lambda(A, H) \rightarrow \Lambda(A, H)$ is a right $\Lambda(A)$ module homomorphism.

We now turn to the generalisation of covariant coordinates.

Proposition 3.3. For any $a \in A$,

$$
L(a) \sigma:=\sigma a-\langle D \sigma, d a\rangle=\sigma a-\langle U \sigma, d a\rangle
$$

is a right module map $L(a): H \rightarrow H$.

Proof. By definition, we have $L(a) \sigma \in H$. To prove that $L(a)$ is a right module homomorphism, we first note that for $b \in A$,

$$
L(a)(\sigma b)=\sigma b a-\langle(D \sigma) b+\sigma d b, d a\rangle .
$$

Now, according to the rules for the q-pairing, we get

$$
\langle(D \sigma) b, d a\rangle=\langle D \sigma, d a\rangle b
$$

and

$$
\langle\sigma d b, d a\rangle=\sigma[b, a] .
$$

Hence $L(a)(\sigma b)=(L(a) \sigma) b$. 
We now show that in the special case of the quantum spacetime algebra $\mathcal{E}$, the $L\left(q^{\mu}\right)$ 's are the covariant coordinates of [12]. For a related discussion, see [16].

Consider the algebra as a module over itself. Pick a covariant derivative, $D a=$ $d a+A a$, where $A$ is a 1 -form. Then for the quantum coordinates $q^{\mu}$, we find

$$
\begin{aligned}
L\left(q^{\mu}\right)(a) & =a q^{\mu}-\left\langle D a, d q^{\mu}\right\rangle=a q^{\mu}-\left\langle d a, d q^{\mu}\right\rangle+\left\langle A a, d q^{\mu}\right\rangle \\
& =a q^{\mu}-\left[a, q^{\mu}\right]+\left\langle A, d q^{\mu}\right\rangle a=q^{\mu} a+\left\langle A, d q^{\mu}\right\rangle a,
\end{aligned}
$$

where we have used the rules (2.11) for the $q$-pairing to pass from the first to the second line. Now, if $A=A_{v}^{(1)} d q^{v} A_{v}^{(2)}$ (in Sweedler's notation), we indeed find,

$$
L\left(q^{\mu}\right)(a)=\left(q^{\mu}+i Q^{\mu v} A_{v}^{(1)} A_{v}^{(2)}\right) a
$$

by application of the rules (2.11).

It should be noted that $L$ is not multiplicative. Instead we have:

Proposition 3.4. Let $a, b, \in A$. Then the algebraic curvature

$$
R_{L}(a, b):=L(a) L(b)-L(a b)
$$

of $L$ is related to the geometric curvature $F=D^{2}$ by

$$
R_{L}(a, b) \sigma=\langle F \sigma, d a d b\rangle
$$

for all $\sigma \in H$.

Proof. Let $D \sigma=\sum \sigma_{i} d c_{i}$. Then

$$
L(b) \sigma=\sigma b-\sum \sigma_{i}\left\langle d c_{i}, d b\right\rangle
$$

and

$$
L(a) L(b) \sigma=(\sigma a-\langle D \sigma, d a\rangle) b-\sum\left(\sigma_{i} a-\left\langle D \sigma_{i}, d a\right\rangle\right)\left\langle d c_{i}, d b\right\rangle .
$$

We have $\sum \sigma_{i} a\left\langle d c_{i}, d b\right\rangle=\sum \sigma_{i}\left\langle d c_{i}, a d b\right\rangle=\langle D \sigma, a d b\rangle$ and $\left\langle D \sigma_{i}, d a\right\rangle\left\langle d c_{i}, d b\right\rangle=$ $\left\langle D^{2} \sigma, d a d b\right\rangle$, hence

$$
L(a) L(b) \sigma=L(a b) \sigma+\langle F \sigma, d a d b\rangle .
$$

Gauge invariant quantities are now obtained in terms of a trace on the algebra of endomorphisms of $H$. For instance, the evaluation of the $a, b$-component of the field strength smeared with a "test function" represented by some suitable element $c$ of $A$ may be defined as

$$
\operatorname{Tr} L(c) R_{L}(a, b)
$$

Therefore, we can interpret the above expressions as providing noncommutative analogues of local gauge invariant quantities. 


\section{The Spectrum of Geometric Operators on Quantum Spacetime}

We will now use the concepts developed in the previous section to define volume, area and distance operators associated with the coordinates $q^{\mu}$ uniquely associated with the quantum spacetime algebra $\mathcal{E}$, as described in the Introduction. To define their spectra we regard the homogeneous elements of the universal differential algebra as elements of tensor powers (of $\mathcal{Z}$-modules) of the quantum spacetime.

We will make use of the noncommutative analogue of the wedge product: For any two tensors $A=\left(A^{\mu_{1} \ldots \mu_{n}}\right), B=\left(B^{\nu_{1} \ldots \nu_{m}}\right)$ with entries from a noncommutative algebra, we define the tensor

$$
A \wedge B=\left(A^{\left[\mu_{1} \ldots \mu_{n}\right.} B^{\left.\nu_{1} \ldots v_{m}\right]}\right),
$$

where the brackets denote total antisymmetrisation as usual. In the highest rank case, we will consider, unless otherwise stated, the component $(A \wedge B)^{0123}$ and denote it (also) by $A \wedge B$.

4.1. The four-volume operator. We start by defining the 4-volume operator as an element of $\Lambda^{4}(\mathcal{E})$,

$$
V=d q \wedge d q \wedge d q \wedge d q=\epsilon_{\mu \nu \rho \sigma} d q^{\mu} d q^{\nu} d q^{\rho} d q^{\sigma},
$$

so that the operator $V$ is an element of the $5^{\text {th }}$ tensor power. It is crucial to observe that, while the product operation used in (4.33) is defined in $\Lambda(\mathcal{E})$, the spectrum of $V$ has to be computed in the completed tensor product $\mathcal{E}^{\otimes 5}$, equipped with its own $\mathrm{C}^{*}$-algebra structure. We interpret $V$ as a function of 5 mutually commuting independent quantum coordinates $q_{j}^{\mu}=1^{\otimes j-1} \otimes q^{\mu} \otimes 1^{\otimes 5-j-1}, j=1, \ldots, 5$, denoting the vertices of a 4 dimensional simplex.

We now represent $V$ as an operator on the $5^{\text {th }}$ tensor power of the representation space of the standard representation and analyse its spectrum. The explicit form of $V$ is

$$
V=\epsilon_{\mu \nu \rho \sigma}\left(q_{2}^{\mu}-q_{1}^{\mu}\right)\left(q_{3}^{\mu}-q_{2}^{\mu}\right)\left(q_{4}^{\mu}-q_{3}^{\mu}\right)\left(q_{5}^{\sigma}-q_{4}^{\sigma}\right) .
$$

We expand this product into a sum of products of coordinates,

$$
V=\sum \pm \epsilon_{\mu \nu \rho \sigma} q_{j_{1}}^{\mu} q_{j_{2}}^{\nu} q_{j_{3}}^{\rho} q_{j_{4}}^{\sigma},
$$

where the sum runs over all choices of $j_{1}, \ldots j_{4} \in\{1, \ldots, 5\}$ with $j_{k} \in\{k, k+1\}$, and where the overall sign is $(-1)^{\sum_{k} j_{k}}$. We may decompose the sum into three parts corresponding to the number of coincidences in the indices $j_{k}$. The only term with 2 coincidences corresponds to

$$
\left(j_{1}, j_{2}, j_{3}, j_{4}\right)=(2,2,4,4) .
$$

This term is

$$
\epsilon_{\mu \nu \rho \sigma} q_{2}^{\mu} q_{2}^{\nu} q_{4}^{\rho} q_{4}^{\sigma}=\frac{1}{4} \epsilon_{\mu \nu \rho \sigma}\left[q_{2}^{\mu}, q_{2}^{\nu}\right]\left[q_{4}^{\rho}, q_{4}^{\sigma}\right] .
$$

We performed the tensor product over the centre, which means that the commutators with different lower indices can be identified, and find

$$
=-\frac{1}{4} Q \wedge Q=-2 \eta,
$$


where $\eta$ is the central element of the algebra $M(\mathcal{E})$ of Quantum Spacetime describing the orientation (with spectrum \pm 1 ).

The part $A$ with no coincidences corresponds to the 5 subsets of $\{1, \ldots, 5\}$ with 4 elements,

$$
A=\sum_{k=1}^{5}(-1)^{k} A_{k}, A_{k}=\bigwedge_{j \neq k} q_{j}
$$

The part with 1 coincidence corresponds to the 10 subsets of $\{1, \ldots, 5\}$ with 2 elements

$$
B=\sum_{i<k}(-1)^{i+k} B_{i k}, B_{i k}=i \frac{1}{2} Q \wedge q_{i} \wedge q_{k}
$$

We obtain the decomposition

$$
V=A+B-2 \eta
$$

Here $A$ and $\eta$ are selfadjoint and $B$ skewadjoint. We now prove that $A$ and $B$ commute, i.e. $V$ is normal.

We have

$$
\left[q_{k}^{\mu}, B_{i j}\right]=Q^{\mu \cdot} \wedge Q \wedge\left(\delta_{i k} q_{j}-\delta_{j k} q_{i}\right)
$$

For any 4-vector $a$ it holds

$$
Q^{\mu \cdot \wedge} \wedge \wedge a=\epsilon_{\nu \lambda \rho \sigma} Q^{\mu \nu} Q^{\lambda \rho} a^{\sigma}=2\left(Q^{\mu \nu}(* Q)_{\nu \sigma}\right) a^{\sigma},
$$

where $* Q$ denotes the Hodge dual of the commutator tensor (1.4b).

But by the relations defining quantum spacetime we have

$$
Q^{\mu \nu}(* Q)_{\nu \sigma}=\frac{1}{4} Q^{\nu \rho}(* Q)_{\nu \rho} \delta^{\mu}{ }_{\sigma}=\eta \delta^{\mu}{ }_{\sigma},
$$

hence we arrive at

$$
\left[q_{k}^{\mu}, B_{i j}\right]=\eta\left(\delta_{i k} q_{j}^{\mu}-\delta_{j k} q_{i}^{\mu}\right)
$$

Thus ad $B_{i j}$ acts on the coordinates $q_{k}$ as $\eta$ times the $i j$-generator of the Lie algebra of $\mathrm{SO}(5)$. It follows that $\operatorname{ad} B$ generates a 1-parameter subgroup of $\mathrm{SO}(5)$. Now

$$
\operatorname{ad} B\left(\sum_{k=1}^{5} q_{k}\right)=\eta \sum_{i<j} \sum_{k}(-1)^{i+j}\left(\delta_{i k} q_{j}-\delta_{j k} q_{i}\right)=\eta \sum_{i<j}(-1)^{i+j}\left(q_{j}-q_{i}\right)=0,
$$

hence this 1-parameter subgroup is contained in the stabiliser $N$ of the vector $(1,1,1,1,1)^{T}$ in the fundamental representation of $\operatorname{SO}(5)$. 
Now $A$ can be written in the form ${ }^{5}$

$$
A=\sum_{j}(-1)^{j} \bigwedge_{i \neq j} q_{i}=\operatorname{det}\left(\begin{array}{ccccc}
1 & q_{1}^{0} & q_{1}^{1} & q_{1}^{2} & q_{1}^{3} \\
1 & q_{2}^{0} & q_{2}^{1} & q_{2}^{2} & q_{2}^{3} \\
1 & q_{3}^{0} & q_{3}^{1} & q_{3}^{2} & q_{3}^{3} \\
1 & q_{4}^{0} & q_{4}^{1} & q_{4}^{2} & q_{4}^{3} \\
1 & q_{5}^{0} & q_{5}^{1} & q_{5}^{2} & q_{5}^{3}
\end{array}\right)
$$

If we multiply the matrix on the right-hand side from the left by a $\mathrm{SO}(5)$-matrix $R$, the left-hand side does not change. If $R \in N$, the right-hand side of (4.47) assumes the form

$$
\operatorname{det}\left(\begin{array}{rrrrr}
1 & q_{1}^{\prime 0} & q_{1}^{\prime 1} & q_{1}^{\prime 2} & q_{1}^{\prime 3} \\
1 & q_{2}^{\prime 0} & q_{2}^{\prime 1} & q_{2}^{\prime 2} & q_{2}^{\prime 3} \\
1 & q_{3}^{\prime 0} & q_{3}^{\prime 1} & q_{3}^{\prime 2} & q_{3}^{\prime 3} \\
1 & q_{4}^{\prime 0} & q_{4}^{\prime 1} & q_{4}^{\prime 2} & q_{4}^{\prime 3} \\
1 & q_{5}^{\prime 0} & q_{5}^{\prime 1} & q_{5}^{\prime 2} & q_{5}^{\prime 3}
\end{array}\right)
$$

where $q^{\prime}{ }_{j}=R_{j}{ }^{k} q_{k}$. Hence $A$ commutes with all linear combinations of $B_{i j}$ corresponding to elements of the Lie algebra of $N$. In particular, $A$ commutes with $B$.

Since $V$ is invariant under proper Lorentz transformations and changes sign under parity, we may evaluate it on a point $\sigma \in \Sigma$ of the spectrum of $Q$. We choose the representation (1.5), where $\sigma=(\vec{e}, \vec{m})$ with $\vec{e}=\vec{m}=(1,0,0)$. We represent the operators $q_{j}^{\mu}$ on the Hilbert space $H^{\otimes 5} \otimes H^{\otimes 5}$ by

$$
\begin{aligned}
& q_{k}^{0}=Q_{k} \otimes I, \\
& q_{k}^{1}=P_{k} \otimes I, \\
& q_{k}^{2}=I \otimes Q_{k}, \\
& q_{k}^{3}=I \otimes P_{k},
\end{aligned}
$$

where the index $k$ refers to the tensor factor in $H^{\otimes 5}$.

In this representation, $A$ assumes the form

$$
\begin{aligned}
A & =\operatorname{det}\left(\begin{array}{lllll}
1 & Q_{1} \otimes I & P_{1} \otimes I & I \otimes Q_{1} & I \otimes P_{1} \\
1 & Q_{2} \otimes I & P_{2} \otimes I & I \otimes Q_{2} & I \otimes P_{2} \\
1 & Q_{3} \otimes I & P_{3} \otimes I & I \otimes Q_{3} & I \otimes P_{3} \\
1 & Q_{4} \otimes I & P_{4} \otimes I & I \otimes Q_{4} & I \otimes P_{4} \\
1 & Q_{5} \otimes I & P_{5} \otimes I & I \otimes Q_{5} & I \otimes P_{5}
\end{array}\right) \\
& =\frac{1}{4} \sum_{i, j, k, l, m=1}^{5} \epsilon_{i j k l m} M_{j k} \otimes M_{l m},
\end{aligned}
$$

where $M_{j k}=Q_{j} P_{k}-Q_{k} P_{j}$ is the generator of rotations in the ( $\left.j k\right)$-plane of $\mathbb{R}^{5}$. We see this by developing first the determinant along the first column, in terms of the

\footnotetext{
5 Note that in our matrices here and in the following only entries on the same row may fail to commute with each other; as a consequence, we can develop indifferently by rows or by columns.
} 
determinants of the $4 \times 4$ complementary minors, and then developing each of those in terms of the determinants of their $2 \times 2$ minors from the two first columns times the determinants of their $2 \times 2$ complementary minors.

We may now multiply the matrix in (4.50) from the left with an $\mathrm{SO}(5)$ matrix, such that the column vector $(1,1,1,1,1)^{T}$ is mapped onto the vector $(0,0,0,0, \sqrt{5})^{T}$. This will not change the operator $A$. The evaluation of the determinant gives

$$
A=\frac{\sqrt{5}}{4} \sum_{j, k, l, m=1}^{4} \epsilon_{j k l m} M_{j k}^{\prime} \otimes M_{l m}^{\prime},
$$

where $M_{j k}^{\prime}$ is the generator of rotations in the $(j k)$-plane in the transformed coordinates, $j, k=1, \ldots, 4$.

Let now $\vec{B}=\left(M_{23}^{\prime}, M_{31}^{\prime}, M_{12}^{\prime}\right), \vec{D}=\left(M_{14}^{\prime}, M_{24}^{\prime}, M_{34}^{\prime}\right)$. Then $\vec{L}^{ \pm}=\frac{1}{2}(\vec{B} \pm \vec{D})$ are mutually commuting generators of SU(2), in terms of which $A$ can be written as

$$
A=2 \sqrt{5}\left(\vec{L}^{+} \otimes \vec{L}^{+}-\vec{L}^{-} \otimes \vec{L}^{-}\right) .
$$

The spectrum of $A$ can now be obtained from an analysis of the representations of $\mathrm{SO}(4)$ in terms of the representations of the two commuting SU(2)'s. They are labeled by pairs of spins $\left(j_{+}, j_{-}\right)$where $j_{+}+j_{-}$, must be integer. We thus obtain

$$
\operatorname{spec}(A) \subset \sqrt{5} \mathbb{Z} .
$$

In view of (4.41) and the fact that $B$ is skewadjoint and commutes with $A$, we find that the spectrum of $V$ has at least a distance $\sqrt{5}-2$ from the origin.

For a full determination of the spectrum of $V$ we now determine the spectrum of $B$. $\eta B$ is a representative of the Lie algebra element

$$
b=\left(\begin{array}{rrrrr}
0 & -1 & 1 & -1 & 1 \\
1 & 0 & -1 & 1 & -1 \\
-1 & 1 & 0 & -1 & 1 \\
1 & -1 & 1 & 0 & -1 \\
-1 & 1 & -1 & 1 & 1
\end{array}\right)
$$

of the Lie algebra $\mathfrak{s o}(5)$ of $\mathrm{SO}(5)$ under the natural representation on $H^{\otimes 5} \otimes H^{\otimes 5}$. The characteristic polynomial of $b$ is

$$
\operatorname{det}(b-\lambda 1)=(-\lambda)^{5}+10(-\lambda)^{3}+5(-\lambda)
$$

with the roots

$$
\lambda_{1}=0, \quad \lambda_{2,3}= \pm i \sqrt{5-2 \sqrt{5}}, \quad \lambda_{4,5}= \pm i \sqrt{5+2 \sqrt{5}} .
$$

We conclude that $B$ has pure point spectrum with eigenvalues of the form $n \lambda_{2}+m \lambda_{4}$ with integers $n$ and $m$. Since $\lambda_{4} / \lambda_{2}=\sqrt{5}+2$ is irrational, the eigenvalues are dense in $i \mathbb{R}$, hence $\operatorname{spec}(B)=i \mathbb{R}$.

The point spectrum of the volume operator $V$ is thus contained in the set

$$
S= \pm 2+\mathbb{Z} \sqrt{5}+i(\mathbb{Z} \sqrt{5-2 \sqrt{5}}+\mathbb{Z} \sqrt{5+2 \sqrt{5}})
$$


It is easy to see that actually all points of $S$ belong to the point spectrum of $V$. Namely, the eigenvalue $a$ of $A$ is determined by fixing the eigenvalues for $\left|\vec{L}^{ \pm} \otimes I\right|^{2},\left|I \otimes \vec{L}^{ \pm}\right|^{2}$ and $\left|\vec{L}^{ \pm} \otimes I+I \otimes \vec{L}^{ \pm}\right|^{2}$. If we call the corresponding spin quantum numbers $j_{1}^{ \pm}, j_{2}^{ \pm}$ and $j_{1}^{ \pm}+j_{2}^{ \pm}-m^{ \pm}$with $m^{ \pm}=0,1, \ldots, 2 \min \left(j_{1}^{ \pm}, j_{2}^{ \pm}\right)$, we obtain

$$
\begin{aligned}
a= & \sqrt{5}\left(\left(j_{1}^{+}+j_{2}^{+}-m^{+}\right)\left(j_{1}^{+}+j_{2}^{+}-m^{+}+1\right)-j_{1}^{+}\left(j_{1}^{+}+1\right)-j_{2}^{+}\left(j_{2}^{+}+1\right)\right. \\
& \left.-\left(j_{1}^{-}+j_{2}^{-}-m^{-}\right)\left(j_{1}^{-}+j_{2}^{-}-m^{-}+1\right)+j_{1}^{-}\left(j_{1}^{-}+1\right)+j_{2}^{-}\left(j_{2}^{-}+1\right)\right) \\
= & \sqrt{5}\left(2 j_{1}^{+} j_{2}^{+}-2 m^{+}\left(j_{1}^{+}+j_{2}^{+}\right)+m^{+}\left(m^{+}-1\right)-2 j_{1}^{-} j_{2}^{-}\right. \\
& \left.+2 m^{-}\left(j_{1}^{-}+j_{2}^{-}\right)-m^{-}\left(m^{-}-1\right)\right) .
\end{aligned}
$$

We now choose $j_{2}^{ \pm}=\frac{1}{2}$. Then $m^{ \pm}=0,1$. We set $m^{ \pm}=0$ and obtain $a=\sqrt{5}\left(j_{1}^{+}-j_{1}^{-}\right)$, thus all integer multiples of $\sqrt{5}$ occur as eigenvalues of $A$.

In order to obtain the eigenvalues of $B$ in a given eigenspace of $A$ we determine the decomposition of the matrix $b$ into the linear combination $b=\alpha_{+} b^{+}+\alpha_{-} b^{-}$of two standard generators $b^{+}, b^{-}$of the two commuting $\mathfrak{s u}(2)$ sub-Lie-algebras in the fundamental representation of $\mathfrak{s o}(4)$ in the 4 dimensional subspace of $\mathbb{R}^{5}$ orthogonal to $(1,1,1,1,1)$. Up to a unitary transformation, these generators are given by

$$
b^{ \pm}=\frac{1}{2}\left(M_{23}^{\prime} \pm M_{14}^{\prime}\right),
$$

and they satisfy the relations

$$
\left(b^{ \pm}\right)^{2}=-\frac{1}{4}, b^{+} b^{-}=b^{-} b^{+}=: \Gamma, \Gamma^{2}=1 .
$$

The coefficients $\alpha_{ \pm}$can be determined from the characteristic equation

$$
b^{4}+10 b^{2}+5=0
$$

Inserting the decomposition of $b$ and using the fact that the even powers of $b$ are linear combinations of 1 and $\Gamma$, we find

$$
\alpha_{+}^{2}+\alpha_{-}^{2}=20
$$

and

$$
\frac{\left(\alpha_{+}^{2}+\alpha_{-}^{2}\right)^{2}}{16}+\frac{\alpha_{+}^{2} \alpha_{-}^{2}}{4}-\frac{5}{2}\left(\alpha_{+}^{2}+\alpha_{-}^{2}\right)+5=0
$$

with the solution

$$
\alpha_{ \pm}^{2}=10 \pm 2 \sqrt{5}
$$

In a representation of $\mathfrak{s o}(4)$ with spin quantum numbers $\left(j^{+}, j^{-}\right)$with $j^{+}+j^{-} \in \mathbb{Z}$ the representative of $b$ thus assumes the eigenvalues

$$
i\left(k^{+} \alpha_{+}+k^{-} \alpha_{-}\right)
$$

with $k^{ \pm}=j^{ \pm}-l^{ \pm}, l^{ \pm}=0,1, \ldots, 2 j^{ \pm}$. In terms of the eigenvalues $\lambda_{2}$ and $\lambda_{4}$ of $b$ (see (4.56),

$$
\lambda_{2,4}=i \sqrt{5 \pm 2 \sqrt{5}}
$$


using

$$
\frac{i}{2}\left(\alpha_{+} \pm \alpha_{-}\right)=\sqrt{5 \pm 2 \sqrt{5}}
$$

we find

$$
i\left(k^{+} \alpha_{+}+k^{-} \alpha_{-}\right)=i\left(k^{+}+k^{-}\right) \lambda_{4}+i\left(k^{+}-k^{-}\right) \lambda_{2} .
$$

We see that every linear combination of $\lambda_{2}$ and $\lambda_{4}$ with integer coefficients can be obtained by an appropriate choice of $j^{ \pm}$and $l^{ \pm}$. We conclude that the set $S$ in (4.57) is the point spectrum of $V$.

The multiplicity of this point spectrum is uniformly infinite. For our operator commutes with the group of joint translations of the five vertices, and the relevant representation of this group has no non zero finite dimensional subrepresentations.

4.2. The three-volume operators. The four components of the 3-volume operator can be expressed, in the formalism used above, as

$$
V_{\sigma}=\epsilon_{\mu \nu \rho \sigma} d q^{\mu} d q^{v} d q^{\rho}=A_{\sigma}+i B_{\sigma}
$$

where

$$
\begin{aligned}
A_{\sigma} & =\frac{1}{6} \operatorname{det}\left(\begin{array}{cccc}
1 & q_{1}^{\mu} & q_{1}^{v} & q_{1}^{\rho} \\
1 & q_{2}^{\mu} & q_{2}^{v} & q_{2}^{\rho} \\
1 & q_{3}^{\mu} & q_{3}^{v} & q_{3}^{\rho} \\
1 & q_{4}^{\mu} & q_{4}^{v} & q_{4}^{\rho}
\end{array}\right) \epsilon_{\mu \nu \rho \sigma}, \\
B_{\sigma} & =\frac{1}{2} Q^{\mu \nu}\left(q_{1}^{\rho}-q_{2}^{\rho}+q_{3}^{\rho}-q_{4}^{\rho}\right) \epsilon_{\mu \nu \rho \sigma} \\
& =\tilde{q}_{1 \sigma}-\tilde{q}_{2 \sigma}+\tilde{q}_{3 \sigma}-\tilde{q}_{4 \sigma} .
\end{aligned}
$$

The operator $V_{\sigma}$ is normal. For, by the Leibniz rule, the commutator of the real with the imaginary part can be computed as $1 / 2$ the sum of the determinants of the 4 by 4 matrices appearing in the last equation, where the $2^{\text {nd }}$, or the $3^{\text {rd }}$, or the $4^{\text {th }}$ column are replaced by the commutators of their entries with $B_{\sigma}$. Thus the first term, for instance, will give

$$
\operatorname{det}\left(\begin{array}{cccc}
1 & {\left[q_{1}^{\mu}, B_{\sigma}\right]} & q_{1}^{v} & q_{1}^{\rho} \\
1 & {\left[q_{2}^{\mu}, B_{\sigma}\right]} & q_{2}^{v} & q_{2}^{\rho} \\
1 & {\left[q_{3}^{\mu}, B_{\sigma}\right]} & q_{3}^{v} & q_{3}^{\rho} \\
1 & {\left[q_{4}^{\mu}, B_{\sigma}\right]} & q_{4}^{v} & q_{4}^{\rho}
\end{array}\right) \epsilon_{\mu \nu \rho \sigma} .
$$

For each $\sigma$ the term $\left[q_{j}^{\mu}, B_{\sigma}\right] \epsilon_{\mu \nu \rho \sigma}$ takes the form

$$
(-1)^{j}\left[q_{j}^{\mu}, \tilde{q}_{j \sigma}\right] \epsilon_{\mu \nu \rho \sigma}=(-1)^{j} \delta^{\mu}{ }_{\sigma} \epsilon_{\mu \nu \rho \sigma}=0 .
$$

Analogous computations can be performed for the other columns, so that our operator is normal. 
It follows that, as operators affiliated to $\mathcal{E}^{\otimes \mathcal{Z}^{4}}$, our $V_{\sigma}$ fulfill

$$
\sum_{\sigma} V_{\sigma}^{*} V_{\sigma}=\sum_{\sigma}\left(A_{\sigma}^{2}+B_{\sigma}^{2}\right) \geqslant \sum_{\sigma} B_{\sigma}^{2}
$$

Now, the $\tilde{q}$ s obey the relations

$$
\left[\tilde{q}_{\sigma}, \tilde{q}_{\rho}\right]=i Q_{\sigma \rho}^{-1},
$$

so that we see by Eq. (4.70b) that the operators $B_{\sigma}$ obey the commutation relations

$$
\left[B_{\sigma}, B_{\rho}\right]=i 4 Q_{\sigma \rho}^{-1} \text {. }
$$

Now, (4.44) shows that $Q^{-1}$ is related to $Q$ by the exchange of $\vec{e}$ and $\vec{m}$, up to a change of sign; therefore the arguments of [1] can be repeated to give

$$
\sum_{\sigma} B_{\sigma}^{2} \geqslant 8
$$

We conclude that the square Euclidean length of the three-volume operator vector is bounded below by 8 in Planck units.

It is worth noting that, however, the spectrum of the time component, namely of the space 3-volume operator, is, as we will show now, the whole complex plane.

That operator can be expressed, in our formalism, as

$$
\begin{aligned}
d \vec{q} \wedge d \vec{q} \wedge d \vec{q} & \equiv \operatorname{det}\left(\begin{array}{cccc}
1 & q_{1}^{1} & q_{1}^{2} & q_{1}^{3} \\
1 & q_{2}^{1} & q_{2}^{2} & q_{2}^{3} \\
1 & q_{3}^{1} & q_{3}^{2} & q_{3}^{3} \\
1 & q_{4}^{1} & q_{4}^{2} & q_{4}^{3}
\end{array}\right)+(i / 2) \sum_{j=1}^{3} m_{j} \sum_{k=1}^{4}(-1)^{k+1} q_{k}^{j} \\
& =A_{0}+i B_{0} .
\end{aligned}
$$

This is a normal operator, whose spectrum will contain the spectrum of the image in any representation; it will then suffice to show that its spectrum is the whole complex plane in the irreducible representation (1.5), where

$$
q^{1}=P \otimes I, q^{2}=I \otimes Q, q^{3}=I \otimes P, \vec{m}=(1,0,0) ;
$$

so that, in that representation, we have

$$
d \vec{q} \wedge d \vec{q} \wedge d \vec{q}=\operatorname{det}\left(\begin{array}{cccc}
1 & P_{1} \otimes I & I \otimes Q_{1} & I \otimes P_{1} \\
\cdot & \cdot & \cdot & \cdot \\
\cdot & \cdot & \cdot & \cdot \\
1 & P_{4} \otimes I & I \otimes Q_{4} & I \otimes P_{4}
\end{array}\right)+(i / 2) \sum_{k=1}^{4}(-1)^{k+1} P_{k} \otimes I .
$$

Furthermore, if we develop the determinant in terms of the determinants of the $2 \times 2$ minors from the two first columns times the determinants of the complementary minors, we find

$$
d \vec{q} \wedge d \vec{q} \wedge d \vec{q}=(1 / 4) \sum \epsilon_{j k l m}\left(P_{k}-P_{j}\right) \otimes M_{l, m}+(i / 2) \sum_{k=1}^{4}(-1)^{k+1} P_{k} \otimes I .
$$


This operator is affiliated to the tensor product of the commutative $C^{*}$-Algebra generated by $P_{1}, \ldots, P_{4}$ with all bounded operators. We can evaluate this operator at a point $p=\left(p_{1}, \ldots, p_{4}\right)$ of the joint spectrum of $P_{1}, \ldots, P_{4}$; re-expressing, as in the previous subsection, the $M_{l, m}$ in terms of the independent generators of two copies of $S U(2)$, we obtain

$$
\mu I \otimes L_{\xi_{+}}^{+}+\lambda I \otimes L_{\xi_{-}}^{-}+i \eta I
$$

where $\xi_{+}, \xi_{-}$are two unit vectors in three space, and $\mu, \lambda, \eta$ are real numbers, all functions of $p$, such that

$$
\begin{gathered}
\mu \xi_{+}=(1 / 2)\left(p_{3}-p_{2}+p_{4}-p_{1}, p_{3}-p_{2}+p_{4}-p_{1}, p_{4}-p_{3}+p_{2}-p_{1}\right) \equiv(u, u, w) ; \\
\lambda \xi_{-}=(1 / 2)\left(p_{4}-p_{3}+p_{2}-p_{1}, p_{1}-p_{3}+p_{4}-p_{2}, p_{1}-p_{3}+p_{4}-p_{2}\right) \equiv(w, v, v) ; \\
\eta(p)=(1 / 2) \sum_{k=1}^{4}(-1)^{k+1} p_{k}=-(1 / 2)\left(p_{4}-p_{3}+p_{2}-p_{1}\right)=-w ;
\end{gathered}
$$

where, as $p=\left(p_{1}, \ldots, p_{4}\right)$ vary in $\mathbb{R}^{4},(u, v, w)$ span $\mathbb{R}^{3}$. Since the joint spectrum of $L_{\xi_{+}}^{+}, L_{\xi_{-}}^{-}$includes all pairs of relative integers, we have that the spectrum of our operators includes the set

$$
\left(2 u^{2}+w^{2}\right)^{1 / 2} \mathbb{Z}+\left(2 v^{2}+w^{2}\right)^{1 / 2} \mathbb{Z}-i w,
$$

for all possible choices of the real numbers $u, v, w$. From these explicit expressions, one sees that the last operator has a spectrum which, as $p$ varies in $\mathbb{R}^{4}$, spans the whole complex plane, and is pure Lebesgue by (4.79).

4.3. The area operators. The area operators can be discussed separately as the spacespace area operator and the space-time area operator, respectively given by the square roots of the sum of the square moduli of

$$
\begin{aligned}
& d q^{j} \wedge d q^{k} \\
& d q^{j} \wedge d q^{0}
\end{aligned}
$$

now,

$$
\begin{aligned}
& \epsilon_{l j k} d q^{j} d q^{k} \\
& \quad=\epsilon_{l j k}\left(I \otimes q^{j}-q^{j} \otimes I\right)\left(I \otimes q^{k}-q^{k} \otimes I\right) \\
& \quad=\epsilon_{l j k}\left(I \otimes q^{j} \otimes q^{k}-q^{j} \otimes I \otimes q^{k}+q^{j} \otimes q^{k} \otimes I\right)+i m_{l} I \otimes I \otimes I,
\end{aligned}
$$

which is a normal operator since $\vec{m}$ is central; the sum over $l=1,2,3$ of the square moduli is then bounded below by $(\vec{m})^{2}$, which is bounded below by $I$ due to the Quantum Conditions.

Quite similarly,

$$
\begin{aligned}
d q^{j} \wedge d q^{0}= & \left(I \otimes q^{j}-q^{j} \otimes I\right)\left(I \otimes q^{0}-q^{0} \otimes I\right) \\
& -\left(I \otimes q^{0}-q^{0} \otimes I\right)\left(I \otimes q^{j}-q^{j} \otimes I\right) \\
= & \left(I \otimes q^{j} \otimes q^{0}-q^{j} \otimes I \otimes q^{0}+q^{j} \otimes q^{0} \otimes I\right) \\
& -\left(I \otimes q^{0} \otimes q^{j}-q^{0} \otimes I \otimes q^{j}+q^{0} \otimes q^{j} \otimes I\right)+i e_{j} I \otimes I \otimes I
\end{aligned}
$$


are normal operators whose sum of the square moduli is bounded below by $(\vec{e})^{2}$, which is bounded below by $I$ due to the Quantum Conditions.

Note that here too normalcy of our operators depends in an essential way upon the fact that the commutators of the coordinates are central.

Thus both the space-space and the space-time area operators are bounded below by 1 in Planck units.

$$
* * *
$$

Finally we would like to point out that we might also have calculated the "dual" length, area, 3- and 4-volume operators, with $q$ replaced by $\tilde{q}=Q^{-1} q$. These yield the contributions to the metric form discussed in Sect. 2. Note that in the case of the highest rank, we find that both $V$ and its dual yield the same contribution to the form, since

$$
d \tilde{q} \wedge d \tilde{q} \wedge d \tilde{q} \wedge d \tilde{q}=\eta d q \wedge d q \wedge d q \wedge d q,
$$

and $\eta$ is the element of $\mathcal{Z}$ equal to plus or minus one on $\Sigma_{+}$or $\Sigma_{-}$respectively. Hence $\eta$ disappears in the square.

\section{Conclusions}

We applied concepts of the universal differential calculus to define several geometric entities on the model of quantum spacetime introduced in [1]. We showed that they can be interpreted in terms of independent events, the underlying classical picture being a characterisation of simplices by their vertices. This makes it possible to represent these entities by operators on a Hilbert space, and we analysed their spectra.

We found that these operators are normal, and that their spectra have an interesting structure, which matches with the general expectations described in the Introduction. Let us comment on the fact that the spectra of these operators are complex. These operators correspond to the volume of simplices in quantum spacetime, and since the vertices of these simplexes are described by noncommuting operators, the permutation of two vertices produces an additional commutator - which of course is skew-symmetric. Hence, the full operator cannot be expected to be self-adjoint and its spectrum will not in general be real. However, the operator's modulus describes the analogue of the classical absolute value of the volume, while the phase is the appropriate generalization of the sign which in classical geometry describes the orientation. We now summarise our findings (where, as above, we adopt Planck units):

1. In the Introduction, we discussed the Euclidean and Minkowskian Distance Operators between two independent events. We proved that, while the second has pure Lebesgue spectrum, the first is bounded below by a constant of order 1, despite the fact that the model is fully Lorentz invariant.

2. The area operator $d q^{\mu} \wedge d q^{\nu}$ can be split into a spatial and a spacetime part. For both the sum of the absolute squares of the components is bounded below by the unit operator.

3. The spectrum of the spatial volume, i.e. the time component of the vector describing the 3-volume operator, is the full complex plane; the sum of the absolute squares of the 4 components is, however, bounded below by 8 .

4. The 4-volume operator has pure point spectrum

$$
\operatorname{spec}_{p p}(V)=S= \pm 2+\mathbb{Z} a_{+} a_{-}+i\left(\mathbb{Z} a_{+}+\mathbb{Z} a_{-}\right),
$$




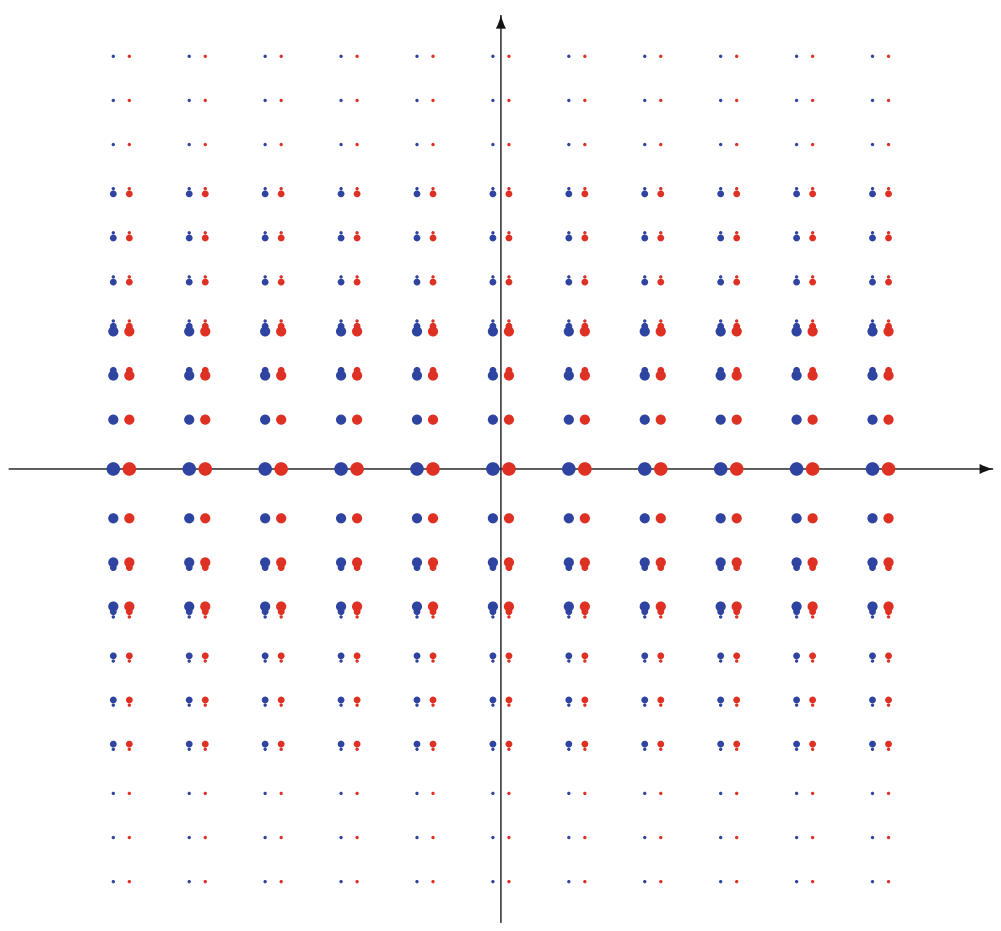

Fig. 1. Generation of the pure point spectrum of the 4-volume operator

where

$$
a_{ \pm}=\sqrt{5 \pm 2 \sqrt{5}}
$$

so that, of course,

$$
\operatorname{spec}(V)=\overline{\operatorname{spec}_{p p}(V)}= \pm 2+\mathbb{Z} \sqrt{5}+i \mathbb{R} .
$$

Figure 1 shows the points in $\operatorname{spec}_{p p}(V)$ with small real part, when the integer coefficients of $a_{+}, a_{-}$have sum of the moduli not exceeding 3 . The left and right sides of each pair of close columns refers to the - and + sign in Eq. (5.81), and larger dots refer to smaller absolute values of the coefficients.

The translates of $S$ by real integers form a ring, so that finite tensor powers and direct sums of copies of $V$ fulfill

$$
\operatorname{spec}_{p p}(V \otimes \cdots \otimes V), \quad \operatorname{spec}_{p p}(V \oplus \cdots \oplus V) \subset \operatorname{spec}_{p p}(V)+\mathbb{Z} .
$$

The combinations of $1, a_{-}, a_{+}, a_{-} a_{+}$with rational coefficients form a field. Presently we have no interpretation of these facts.

We have seen that the appropriate mathematical frame to establish these results is a combination of the universal differential calculus on one side and the relevant $\mathrm{C}^{*}$-algebraic structures on the other.

This mathematical frame allowed us to recognise the Hochschild boundary as a Hodge dual of the absolute differential. This duality arises from a "quantum pairing" which vanishes exactly in the commutative case. Nevertheless we used this pairing to 
introduce a metric which generalises the classical metric, and which would allow us to define noncommutative analogues of an action. In its present form, however, this approach is valid only in a flat background.

Moreover, applying this formalism to projective modules, we gave a definition of parallel transport in terms of connections, and we introduced a linear homomorphism from the algebra to the endomorphisms of our right module, whose algebraic curvature is related to the geometric curvature. This makes it possible to introduce local gauge invariant quantities; in the case of our quantum spacetime, this homomorphism maps the coordinates to the covariant coordinates of [12].

Acknowledgements. We are pleased to thank Joachim Cuntz and Jochen Zahn for helpful discussions and comments. S.D. gratefully acknowledges the support received from the Alexander von Humboldt Foundation in various stages of this research. D.B. was supported by the German Research Foundation (Deutsche Forschungsgemeinschaft (DFG)) through the Institutional Strategy of the University of Göttingen.

Open Access This article is distributed under the terms of the Creative Commons Attribution Noncommercial License which permits any noncommercial use, distribution, and reproduction in any medium, provided the original author(s) and source are credited.

\section{References}

1. Doplicher, S., Fredenhagen, K., Roberts, J.E.: The Quantum structure of space-time at the Planck scale and quantum fields. Commun. Math. Phys. 172, 187-220 (1995)

2. Amati, D., Ciafaloni, M., Veneziano, G.: Higher order gravitational deflection and soft bremsstrahlung in Planckian energy superstring collisions. Nucl. Phys. B347, 550-580 (1990)

3. Mead, C.A.: Possible Connection Between Gravitation and Fundamental Length. Phys. Rev. 135, B849B862 (1964)

4. Doplicher, S., Fredenhagen, K., Roberts, J.E.: Space-time quantization induced by classical gravity. Phys. Lett. B331, 39-44 (1994)

5. Bahns, D., Doplicher, S., Fredenhagen, K., Piacitelli, G.: Ultraviolet Finite Quantum Field Theory on Quantum Spacetime. Commun. Math. Phys. 237, 221-241 (2003)

6. Doplicher, S.: "Spacetime and fields, a quantum texture." In: New Developments in Fundamental Interaction Theories: $37^{\text {th }}$ Karpacz Winter School of Theoretical Physics, J. Lukierski, J. Rembielinski, eds., Melville, NY: AIP, 2001, pp. 204-213

7. Cuntz, J.: "Cyclic theory and the bivariant chern-connes character." In: Noncommutative Geometry, S. Doplicher, R. Longo, eds., Berlin Heidelberg-New York: Springer, 2004, pp. 73-135

8. Connes, A.: Noncommutative Geometry. London-New York: Academic Press, 1994

9. Dubois-Violette, M.: Dérivations et calcul differentiel non commutatif. C. R. Acad. Sci. Paris. 307, 403408 (1988)

10. Cuntz, J.: Private communication

11. Gracia-Bondia, J. M., Varilly, J. C., Figueroa, H.: Elements of noncommutative geometry. Bosel-Boston: Birkhäuser, 2001

12. Madore, J., Schraml, S., Schupp, P., Wess, J.: Gauge theory on noncommutative spaces. Eur. Phys. J. C16, 161-167 (2000)

13. Fredenhagen, K.: "Quantum Fields and Noncommutative Spacetime.” In: Noncommutative Geometry and the Standard Model of Elementary Particle Physics. Proceedings of the Hesselberg Conference, March 14-19, 1999, F. Scheck, W. Werner, H. Upmeier, eds., Berlin Heidelberg-New York: Springer-Verlag, 2002, pp. 282-289

14. Dimakis, A., Muller-Hoissen, F., Striker, T.: Noncommutative differential calculus and lattice gauge theory. J. Phys. A. Gen. 26, 1927-1949 (1993)

15. Prüstel, T.: Gauge Theories with Nonunitary Parallel Transport. PhD thesis, University of Hamburg, 2003

16. Zahn, J. W.: Dispersion relations in quantum electrodynamics on the noncommutative Minkowski space. $\mathrm{PhD}$ thesis, University of Hamburg, 2006, http://arxiv.org/abs/0707.2149v1 [hep-th], 2007

Communicated by M. Salmhofer 